\title{
Quando il popolo va in scena. Arti, mestieri e professioni nei teatri minori del Settecento
}

Paola Martinuzzi

\section{(2) OpenEdition}

\section{Journals}

\section{Edizione digitale}

URL: http://journals.openedition.org/studifrancesi/5206

DOI: $10.4000 /$ studifrancesi. 5206

ISSN: 2421-5856

\section{Editore}

Rosenberg \& Sellier

\section{Edizione cartacea}

Data di pubblicazione: 1 dicembre 2016

Paginazione: 424-434

ISSN: 0039-2944

\section{Notizia bibliografica digitale}

Paola Martinuzzi, «Quando il popolo va in scena. Arti, mestieri e professioni nei teatri minori del Settecento », Studi Francesi [Online], 180 (LX | III) | 2016, online dal 01 janvier 2018, consultato il 18 septembre 2020. URL : http://journals.openedition.org/studifrancesi/5206 ; DOI : https://doi.org/ 10.4000/studifrancesi.5206

\section{(c) (i) (9)}

Studi Francesi è distribuita con Licenza Creative Commons Attribuzione - Non commerciale - Non opere derivate 4.0 Internazionale. 


\title{
Quando il popolo va in scena. Arti, mestieri e professioni nei teatri minori del Settecento
}

\begin{abstract}
Although, between $17^{\text {th }}$ and $18^{\text {th }}$ Century, popular culture was repressed and submitted to dominant culture in France, minor theatres tended to merge low and high, folk atemporal elements and literary references. The public going to fair theatres was made up by people coming from different classes and this fact encouraged the artists to create comic plays where the two cultures could meet, in a new conception of theatre. This article focuses on the presence of craftsmen, ambulant workers, farm-hands in Parisian fair theatres dramaturgy and performance, during the first half of the $18^{\text {th }}$ Century. These figures are not conceived as decorative elements but they are put at the heart of the plays. There are several ways of combining popular and élite culture, in performances founded on wonder, in parodies of tragedies and operas or by giving new dignity to humble people by underlining the value of art and industrie, in accordance with Diderot's ideas originating the Encyclopédie, and also by proposing their language and argot as a mean to enrich literary style, as in poissard production.
\end{abstract}

È durante il regno di Luigi XIV che si assiste, secondo Robert Muchembled, al tramonto, anzi a una vera e propria repressione del sapere popolare e contemporaneamente alla nascita della cultura di massa, ovvero di una cultura dipendente da quella dotta e della quale assume i riflessi ${ }^{1}$. Lo storico cita Norbert Elias, che aveva messo a fuoco il distacco e la differenziazione dei due mondi sociali e la conseguenza di questo fenomeno, ossia la divulgazione degli ideali e della morale "dominanti" e delle forme letterarie ad essi connesse, con una svalutazione della cultura popolare 2 . Questo lungo processo, il cui percorso coincide con l'affermazione del centralismo politico e religioso, condusse singolarmente alla produzione di libri, opuscoli e stampe di larga diffusione intesi a "educare" la popolazione e a convogliare lo spirito arcaico e la superstizione nella corrente ordinata del culto e della storia nazionale.

La separazione tra le due culture ha portato in molti casi, fra Seicento e primo Settecento, a utilizzare nei teatri "maggiori" le figure popolari in chiave burlesca, come espressione di quel gusto per il concreto e per il «pittoresque de la vie» che Paul Hazard collocava a pieno titolo, accanto al dépaysement, all'esotismo, al meraviglioso, nella crise de la conscience européenne $e^{4}$. Questa scelta era strettamente connessa con la

(1) R. Muchembled, Culture populaire et culture des élites dans la France moderne (XV'-XVIII siècles), Paris, Flammarion, 1978.

(2) N. ELIAS, Il processo di civilizzazione (1939), tr. it. di G. Panzieri, Bologna, il Mulino, 1988, 2 voll.

(3) $\mathrm{Ci}$ riferiamo naturalmente alla Bibliothèque bleue edita a Troyes; cfr. a riguardo, gli studi di G. Bollème, Les Almanachs populaires aux XVII et XVIII' siècles, essai d'histoire sociale, Paris-La Haye, Mouton, 1969; D. Roche, Le peuple de Paris, Paris,
Éditions Aubier Montaigne, 1981; R. MANDROU, De la culture populaire aux XVII' et XVIII' siècles, Paris, Éditions Imago, 1985; L. AndRIÈs, La Bibliothèque bleue au XVIII siècle, Oxford, Voltaire Foundation, 1989; ID., Le grand livre des secrets: le colportage en France aux XVII et XVIII siècles, Paris, Éditions Imago, 1994.

(4) Per questo concetto, cfr. P. Hazard, La crise de la conscience européenne (1680-1715), t. II, Paris, Boivin et Cie Éditeurs, 1935, in particolare pp. 181194. 
fruizione dello spettacolo da parte di un pubblico essenzialmente aristocratico; non vi era spazio per una adesione morale o emotiva alle immagini messe in scena, ma emergeva la distanza, che si traduceva in un riso ironico o nel travestimento dello spirito galante nelle vesti di piccoli e curiosi personaggi. E possiamo vederne degli esempi anche in momenti più remoti, come nel balletto di corte burlesco, dove il personaggio ricorrente del ciarlatano dava espressione ai «ricanements cyniques» dell'«esprit frondeur» ${ }^{5}$ o dove gli artigiani, come il maniscalco, il tessitore e mestieranti come lo spazzacamino non facevano che riproporre in false vesti popolari la retorica del tema amoroso $^{6}$.

Vedremo qui che sulle scene non istituzionali della prima metà del secolo, frequentate da un pubblico misto, non vi è questo dislivello fra mondo rappresentato e destinatari della rappresentazione; la realtà quotidiana, pur nell'ambito dei generi comici, può godere di una nuova dignità. L'intento non è però quello perseguito da alcune opere realistiche coeve, come i celebri romanzi di Marivaux, in cui le figure umili vivono un percorso che le conduce a un rango sociale di maggiore considerazione, percorso che appare anche in opere teatrali di Nivelle de La Chaussée e di LouisSébastien Mercier. Ciò che conta, nei teatri marginali rispetto ai centri politici, ma innovatori come quelli delle Fiere parigine, è offrire una immagine viva e dinamica del mondo, il più delle volte per tableaux, in esposizioni brevi; e ogni volta che vi è caricatura, essa non risparmia nessuno, dal mugnaio astuto agli alti funzionari corrotti.

Il repertorio delle pièces create alla Foire Saint-Germain e alla Foire Saint-Laurent in quasi novant'anni di attività (dalle prime notizie pubblicate del 1678, alla fusione dell'Opéra Comique con la Comédie Italienne, nel 1762$)^{7}$, supera il migliaio di titoli; ne fanno fede il repertorio di Brenner e il Calendrier Électronique des Spectacles sous l'Ancien Régime 8 . Per molte commedie', il testo si è perduto, le testimonianze sono disomogenee; si tratta di opere spesso rappresentate anche solo una volta, in un incessante dialogo fra le attese del pubblico e gli sforzi degli artisti per continuare a lavorare e produrre novità, anche quando il controllo dell'autorità politica, di concerto con i suoi organi culturali rappresentati dai teatri finanziati dal sovrano, sembrava impedire ogni iniziativa esterna al monopolio istituito con Jean-Baptiste Lully ${ }^{10}$.

Proprio la situazione di indipendenza nella gestione e programmazione dei teatri e il ruolo storicamente avuto dalle Fiere, di punto d'incontro fra persone di varia provenienza geografica e sociale, hanno favorito, in modo spontaneo e non programmatico, una prospettiva moderna di avvicinamento e non di scissione fra le due culture. La coesistenza di un immaginario e di uno spirito arcaici e folklorici, insieme a rimandi letterari e artistici di una grande varietà, comprensibili interamente da parte

(5) G. Durosorr, "Je suis le fameux empirique". Opérateurs, empiriques et autres charlatans dans les ballets de cour au temps de Louis XIII, «Théâtre et drame musical» 3-4, 2004, p. 30.

(6) Cfr. Le Ballet des mestiers (1633 ca.), in Mascarades et ballets de cour de Henri III (1581-1652), éd. P. Lacroix, t. IV, Genève-Turin, J. Gay et fils, 1868-1870, pp. 287-291.

(7) Il 1762 segna anche un momento difficile per il grosso incendio che distrusse la Foire Saint-Germain e a cui seguirono la ricostruzione e un nuovo assetto.

(8) C.D. Brenner, A Bibliographical List of Plays in the French Language, 1700-1789 (1947), New York, AMS Press, 1979; CESAR, Calendrier Électronique des Spectacles sous l'Ancien Régime et la Révolution, http://cesar.org.uk/cesar2/index.php.
(9) Il termine, anche se utile, non è corretto, perché la varietà dei generi e la presenza pressoché continua della musica e del canto impediscono una assimilazione al modello con cui la commedia si è definita, nelle culture europee.

(10) E noto che la fondazione da parte di Luigi XIV dell'Académie Royale de Musique, nel 1669, fu accompagnata dalla formulazione, con lettere patenti, del privilegio di rappresentarvi in modo esclusivo opere in musica e in versi francesi, nella capitale. Alcune imprese teatrali attive negli spazi delle fiere parigine acquisirono, dal 1715 al 1745, a titolo oneroso il privilegio, da dover comunque rinnovare, con danno economico e sempre nella precarietà data dai decreti iterati. Questo patto dette vita al teatro dell'Opéra Comique. 
di spettatori letterati o frequentatori assidui dei vari teatri, costituisce l'originalità del teatro forain, in particolare nella prima parte del Settecento. L'incontro più evidente fra alto e basso è nelle opere in cui prevale l'elemento del merveilleux, ma esso si realizza anche in altre tipologie.

Procedendo verso la metà del secolo, si è andato imponendo un gusto borghese che ha determinato in molte occasioni una aderenza alla bienséance, lo sviluppo di intrecci sentimentali non puramente schematici e una selezione di argomenti collegati alla vita economica e familiare. Esempio ne sono numerosi opéras-comiques di Favart, spesso ambientati in un villaggio, in cui sono coinvolti, con ruoli non marginali, ussari e maréchaux des logis ${ }^{11}$, notai ${ }^{12}$, procureurs fiscaux ${ }^{13}$, balivi ${ }^{14}$. D'altra parte, come lo storico Maurice Albert lamenta, si impone attorno al 1754 un'altra voga, inaugurata da Jean-Joseph Vadé, quella del genere poissard; in esso lo spettacolo si sbilancia verso una comicità essenzialmente verbale e grossolana che cancella i virtuosismi fisici attoriali e il non-sens ereditati dalla Commedia dell'Arte, proponendo così un mondo popolare non più connesso con i terreni arcaici delle maschere. Va approfondendosi un percorso già iniziato nei decenni precedenti, che avevano visto quella che Claudio Vinti definisce come perdita della «essenzialità tipologica quasi atemporale» delle maschere $^{15}$; scompaiono i tipi fissi ereditati dai comici italiani e si moltiplicano i personaggi i cui caratteri vengono di volta in volta assunti dagli attori, senza fondersi con la personalità artistica degli interpreti. In Vadé pullulano i mestieri con il linguaggio desunto dalla strada e dalla taverna, l'argot.

Pour quelques années la place appartient aux personnages populaires dont le poète est allé poursuivre les modèles dans tous les carrefours dans la banlieue, même au fond des campagnes normandes. Sur la scène où voltigeaient jadis le svelte Arlequin, l'aimable Pierrot et la gracieuse Colombine, aujourd'hui se campent lourdement des marchandes de marée, des pêcheurs de goujons, des blanchisseuses, des forts de la Halle, des maraîchers et des paysans, des soldats et des perruquiers ${ }^{16}$.

Secondo Albert, che considera come maggioritario ma non esclusivo un pubblico borghese, il gergo popolare di queste figure «ne dut pas, à l'origine, moins ahurir le bourgeois que le jargon médité des Précieuses» ${ }^{17}$; nello stesso tempo allo scrittore è riconosciuto l'intento rinnovatore di mettere in scena un linguaggio e una dizione triviali, gesti brutali ma dal carattere «naturel, franc, joyeux et coloré» ${ }^{18}$. Fra gli spettatori dell'epoca, il marchese d'Argenson, abitualmente scettico e sprezzante verso il comico basso, in questo caso apprezza sinceramente lo studio della lingua del volgo e dei porti, affrontato da Vadé «profondément et constament»». La letteratura poissar-

(11) Ch.-S. Favart, Moulinet premier, Parodie de Mabomet second, La Haye, van Dole, 1739. I maréchaux des logis avevano il compito di precedere il seguito del Re e di contrassegnare con il gesso gli alloggi dove avrebbero preso posto i membri della corte. Il protagonista di questo opéra-comique è Moulinet, comandante di un reggimento di ussari che funge da corrispettivo parodico dell'imperatore turco, eroe di La Noue nella tragedia Mabomet second (1739) ambientata a Bisanzio.

(12) Cfr. ID., Le Coq de village (1743), Paris, Prault, 1744. Si tratta, qui come in altre pièces, di un notaio di campagna, il tabellion.

(13) Cfr. ID., La Coquette sans le savoir (1744), Paris, Prault, 1744 (con musica di Antoine Dauvergne). I procureurs fiscaux si occupavano dell'am- ministrazione dei beni di notabili dotati di autorità giuridica.

(14) Cfr. ID., L'Amour au village (1745), Paris, Delormel-Prault, 1752.

(15) C. VINTI, Alla foire e dintorni. Saggi di drammaturgia foraine, Roma, Edizioni di Storia e Letteratura, 1990, p. 22.

(16) M. AlBERT, Les théâtres de la Foire (16601789), Paris, Hachette, 1900 (Genève, Slatkine, 1969), p. 201.

(17) Ivi, p. 202

(18) Ivi, pp. 204-205.

(19) R.-L. DE Voyer D'Argenson, Notices sur les Euvres de théâtre, publiées par H. Lagrave, Genève, Droz, 1966, «Studies on Voltaire and the Eighteenth Century», v. XLIII, p. 597. 
de, per d'Argenson, non rappresenta un divertimento ironico, ma si rivela un modo di conoscenza della realtà a tutti gli effetti, una «science» che diletta l'intero pubblico, «mêsme la bonne compagnie (car elle se raproche de cet idiome par la conversation avec les courtisanes) $\gg^{20}$.

In effetti, tra gli spettatori dell'Opéra Comique durante gli anni Cinquanta non vi era più la servitù, per effetto di un'ordinanza del 21 gennaio $1745^{21}$, che l'aveva esclusa per ragioni d'ordine pubblico, ma la composizione del parterre continuava ad essere varia, come sostiene Henri Lagrave ${ }^{22}$. Aristocratici, religiosi, borghesi, studenti, artigiani e venditori (piccoli e grandi) frequentavano i teatri attivi nei luoghi del commercio. Questi elementi ci portano a credere che la rappresentazione del popolo sulle scene della Foire fosse il risultato di una intesa alla base, fra artisti e destinatari dello spettacolo, che non portava a giudicare dall'alto.

Rosalba Gasparro ha rilevato le difficoltà e il rischio affrontati dai teatri della Foire nel rappresentare l'«antisublime», il basso, il quotidiano attraverso la degradazione del linguaggio ${ }^{23}$. D'altronde, una trasformazione di tutto il repertorio forain in questa direzione avrebbe portato alla monotonia e il direttore Jean Monnet, che pure era catturato dal «génie» di Vadé ${ }^{4}$, al punto da lasciare il proprio incarico alla morte del drammaturgo, continuò a programmare contemporaneamente anche degli opérascomiques parodici e dei ballets-pantomimes.

Ci soffermiamo sulla presenza dei mestieri in una scelta di opere che sono espressione di un teatro rivolto a un pubblico misto e non elitario. Consideriamo per prime le figure sociali che esercitavano la loro professione nelle strade, unendo alla realizzazione del lavoro specifico la performance, veri momenti di spettacolo che assolvevano una funzione di propaganda per la propria attività. Ciarlatani, imbonitori, chirurghi non appartenevano ad alcuna corporazione, molto più simili a vagabondi che a professionisti; esibivano animali ammaestrati e mantenevano vivo il patrimonio drammatico della farsa con i suoi tópoi, alimentavano la ricchezza della lingua e perpetuavano in tono minore l'arte della retorica. Il teatro se ne appropria nei suoi vari contesti e dà una valenza critica a questa metateatralità che porta sul palcoscenico una scena più piccola desunta dalla piazza.

Il linguaggio dell'Opérateur, che oscilla mirabilmente fra i significati metaforici e quelli letterali, può richiamare buffonescamente l'enfasi delle orazioni morali. Tra i suoi tanti rimedi egli vanta un «tabac preparé pour tirer les vers du nez», ossia per espungere con astuzia i segreti; altrettanto prodigiosa è «la véritable poudre de corail pour ceux qui saignent du nez quand il s'agit de remplir leurs promesses». Si tratta di una polvere miracolosa che non solo, come vuole la superstizione, allontana gli spiriti maligni e protegge le abitazioni dai fulmini, ma fa mantenere le promesse date ${ }^{25}$.

L'Empirique può anche entrare in scena accompagnato dal suo assistente, il Frater (come era scherzosamente chiamata la figura dell'apprendista chirurgo a partire dai primi decenni del secolo), e agitando nell' aria un bisturi, bisticciando futilmente con lui, nella prosecuzione del non-sens, può vedersi consegnare, da una Porteuse d'eau, un secchio pieno d'acqua da distribuire. L'umile venditrice ambulante, presen-

(20) Ibid.

(21) Cfr. J. Monnet, Mémoires de Jean Monnet, directeur du Théâtre de la Foire, introduction et notes par H. D’Alméras, Paris, Louis-Michaud, [1908 secondo Auguste Rondel], nota a p. 79.

(22) H. Lagrave, Le théatre et le public à Paris de 1715 à 1750, Paris, Librairie Klincksieck, 1972.

(23) R. GASPARRo, Il viaggio della maschera: tipologie del comico da Moliere alla Foire, Messina,
I.B.H., 1988, pp. 60-61.

(24) J. MONNET, op. cit., p. 165.

(25) Cfr. A. Furetière, Dictionnaire universel, La Haye, Pierre Husson, 1727 e Ph.-J. LE Roux, Dictionnaire comique, satyrique, critique, burlesque, libre et proverbial, Amsterdam, Zacharie Chastelain, 1750. La citazione è da Les Rivaux de village di Ch.-F. PANnARd (1738-39), BnF, ms. f. fr. 9324 , f. $143 \mathrm{v}$. 
za familiare e necessaria nelle vie cittadine e memoria delle farse ${ }^{26}$, può emergere allora in modo del tutto soprannaturale da un'urna, evocata come se fosse un bigliettino della buona sorte, da Harpocrate, il dio del silenzio nei misteri dionisiaci che i forains hanno eletto, negli anni difficili, a loro protettore ${ }^{27}$.

In questi esempi desunti da Pannard, il "basso" tende a inglobare sia lo stile letterario che la citazione colta e da tale fusione si genera un effetto poetico di straniamento. Come ha rilevato Nathalie Rizzoni, «Pannard s'est employé tranquillement à démontrer par la pratique - c'est-à-dire à travers sa propre production - que le théâtre est un moyen incomparable d'instruction morale et esthétique pour le plus grand nombre ${ }^{28}$. Utilizzando i termini di Jauss e applicandoli allo spettacolo, potremmo dire che l'esperienza maturata dallo spettatore e l'arricchimento che deriva da questa esperienza si possono realizzare «anche contro lo sfondo dell'esperienza quotidiana $^{29}$; non si tratta di un teatro che riflette soltanto la realtà e le attese del pubblico borghese.

La venditrice d'acqua che sostiene sulle sue spalle il peso dei secchi ${ }^{30}$ appartiene alla lunga serie di petits métiers che dalla realtà quotidiana urbana scorre sulla scena portandovi un respiro vitale. Compaiono le medesime figure ambulanti che si vedono incise nei Cris de Paris: Ravaudeuses, Cordonniers, Chaudronniers, Remouleurs (o Gagne-petit), Marchands de chansons, Savoyards, Portefaix, Écaillers, Harengères, Bouquetières, Marchandes de tisane. I venditori di tisane si impongono con il cri di «À boire, à boire, à boire!» ${ }^{31}$, che risuona come il rintocco di una campana mentre avanzano e lodano la bevanda: «Elle n'ote jamais ny raison ny memoire» ${ }^{32}$, e «Celui-ci ne vous montera pas à la tête» ${ }^{33}$. Ma questa virtù è rifiutata buffonescamente con colpi di alabarda da parte di uno Svizzero, tipo fisso dell'avvinazzato nel teatro comico: «D'ein coup de pertissane | Percir tripe et boudin $»^{34}$. Frammenti di realtà si confrontano con la finzione e con citazioni dalla letteratura teatrale; lo spirito acceso polemico e la pronuncia parodiata dello Svizzero richiamano infatti l'analogo personaggio di Molière nel Ballet des Nations del Bourgeois gentilhomme.

Accanto a questi ambulanti, è facile che vi siano i Savoyards. Fra essi, i personaggi maschili illustrano con eloquenza e spirito critico le immagini che proiettano nella lanterna magica, avvicinano gli spettatori con richiami così concepiti: «la rareta, la curiosita ${ }^{35}$,

(26) Cfr. Farce plaisante et recréative sur un trait qu'a joué un porteur d'eau le jour de ses nopces dans Paris, in Le Théâtre Français avant la Renaissance: 1450-1550, éd. É. Fournier, Paris, Laplace, Sanchez, 1880 , pp. 456-460. Il tono realistico della farsa non è ripreso dai forains, più sensibili al confronto inatteso fra stili contrastanti.

(27) Ch.-F. PANNARD, Les Oracles d'Harpocrate, ou le Dieu du Silence à la Foire, pantomime, Paris, Veuve Delormel, 1746, pp. 2-3. Questa singolarissima opera metateatrale funge, come numerose altre nel corso dei decenni, da requisitoria contro i teatri ufficiali, dopo la chiusura dell'Opéra Comique nel 1745, stremata dal prezzo sempre più alto richiesto dall'Académie Royale de Musique per acquisire il privilegio.

(28) N. Rizzoni, Marivaux, Pannard et "Le Chemin de fortune", «Revue d'Histoire Littéraire de la France» XCII, 2, mars-avril 1998, p. 220.

(29) H.R. JAUSs, Literaturgeschichte als Provokation (1967), citato da R. WEImann, Estetica della ricezione, in Teoria della ricezione, dir. R.C. HoLuB, Torino, Einaudi, 1989, p. 96.

(30) Un Porteur e una Porteuse d'eau appaiono accanto a un Soldat e a una Grivoise (una vivandiera) nella danza de Les Désesperés di Lesage e D'Orneval (1732), in A.-R. Lesage, J.-Ph. D'ORneval, Le Théâtre de la Foire ou l'Opéra Comique. Contenant les meilleures pièces qui ont été représentées aux Foires de Saint-Germain et de Saint-Laurent, t. IX, Paris, Gandouin, 1737, pp. 116-152.

(31) J.-Ph. D'Orneval, Ch.-F. Pannard, A.-R. Lesage, L'Impromptu du Pont-Neuf (1729), in A.-R. Lesage, J.-Ph. D'Orneval, Le Théâtre de la Foire ou l'Opéra Comique cit., t. VII, p. 305; Ch.-F. PANNARD, Ch.-S. FAVART, Scenes détachées pour couper la danse dans le balet de la Foire de Bezons (1735), BnF, ms. f. fr. 9325, f. 140r, in P. MarTinuzzi, Ironie della lanterna magica ( LLa Foire de Bezons», ballettopantomima inedito), «Ariel» XXIII, 2, 2008, p. 69.

(32) Ch.-F. Pannard, Ch.-S. Favart, La Foire de Bezons cit., f. 140r., ibid.

(33) J.-Ph. D’Orneval, Ch.-F. Pannard, A.-R. LeSAgE, L'Impromptu du Pont-Neuf cit., p. 306.

(34) Ibid.

(35) Ch.-F. Pannard, Ch.-S. Favart, La Foire de Bezons cit., ff. 143r-145r, ed. cit., pp. 73-76. 
«Ah! qui veut voir la piéce mirlifique!» ${ }^{36}$; le donne e i bambini attirano l'attenzione dei passanti esibendo le marmotte custodite in cassette rettangolari che portano a tracolla e gridano: «Ah! la petite marmotte en vie qui danse toute seule» ${ }^{37}$. Anche in questo caso, come per il ciarlatano, la scena metateatrale è indirizzata verso la satira delle cose e dei fatti che mostra la lanterna, con un movimento contrario alla fiduciosa meraviglia che questi spettacoli ottici normalmente suscitavano.

Vari pescivendoli compaiono sulle scene della Foire, e anche pescatori (collocati questi in uno spazio non urbano). In questo gruppo, sono ricorrenti le Harangères, le venditrici di aringhe fresche o salate che per il loro vociare caratteristico e sguaiato hanno dato vita a espressioni idiomatiche della lingua francese come è attestato dal $1656^{38}$, ma già François Villon ne aveva immortalato la parlantina nella Ballade des femmes de Paris ${ }^{39}$. Una di esse, in Pannard, si lamenta con amarezza del marito che consuma tutto ciò che lei guadagna ${ }^{40}$; un'altra minaccia di schiaffeggiare un procuratore restio a festeggiare la nascita del Delfino, evento festivo che coinvolge nello spazio pubblico tutti i ceti sociali: «Sçais-tu bien que je t'appliquerai une giroflée à cinq feüilles» ${ }^{41}$. Nella parodia della tragédie-lyrique Thésée, un coro di Harangères accompagna il corteo del bue grasso e Teseo, cantando «Gué, gué, gué opégué» e danzando in tondo, al posto del popolo di Atene esultante $^{42}$; con il loro linguaggio ridondante di «liaisons abusives», denigrano Thésée, chiamandolo «zéros» $[$ sic $]$ e non «héros» ${ }^{43}$. Non è specificato nei libretti se questi personaggi vendessero pesce appena pescato o conservato; nell'organizzazione delle attività commerciali, nel primo caso le venditrici appartenevano al corpo dei «poissonniers de mer», nel secondo, a quello dei «marchands de salines», un commercio giunto a Parigi dal XII secolo ${ }^{44}$. Le aringhe costituivano l'unico cibo proteinico di una gran parte della popolazione ${ }^{45}$; le Harangères a teatro fungono da personaggi portatori di caratteri spiccatamente popolari e conservano la litigiosità delle venditrici reali sia di pesci che di fiori, testimoniata dai viaggiatori del Settecento ${ }^{46}$.

Diverso è lo scenario reale per i venditori di ostriche, settore che godeva di un privilegio e di una regolamentazione dell'allevamento, della pesca e del commercio

(36) L. Anseaume, Ch.-S. Favart, Le Boulevard, opéra-comique, ballet, Paris, Duchesne, 1753, p. 20.

(37) Ivi, p. 21. La sensibilità degli artisti è stata toccata in molte occasioni da questi ambulanti, lo dimostrano fra gli altri i dipinti e le incisioni settecenteschi di Antoine Watteau, di François Boucher, di Francesco Maggiotto.

(38) Dictionnaire bistorique de la langue française, sous la direction d'A. Rey, Paris, Dictionnaires Le Robert, 1993.

(39) «Brettes, Suysses, n’y sçavent guieres, I Gasconnes, n'aussi Toulousaines: I De Petit Pont deux harengieres | Les concluront». F. VILLON, Ballade des femmes de Paris, in Opere, ed. E. STOJKovic Mazzariol, Milano, Mondadori, 1990, p. 160.

(40) Ch.-F. Pannard, L'Industrie, Balet mêlé de sçenes (1737), BnF, ms. f. fr. 9323, f. 426v.

(41) J.-Ph. D’Orneval, Ch.-F. Pannard, A.-R. Lesage, L'Impromptu du Pont-Neuf cit., p. 310. Questo spettacolo. che propone una galleria di personaggi desunti dalla vita cittadina, fu rappresentato gratuitamente alla Foire Saint-Laurent il 9 settembre 1729 in occasione della nascita dell'atteso figlio maschio di Luigi XV. L'interesse della pièce è nella serie rapida di figure che compone una parade; vi sono anche un gruppo di Bouquetières, una Savoyarde con la marmotta e persino il celebre cavadenti Grand-Thomas.

(42) Ch.-S. Favart, P. Laujon, Parvi, Thésée, parodie nouvelle de Thésée [de Quinault-Lully] (1745), Paris, Prault-Delormel, 1745, p. 18. Cfr. Ph. Quinault, Thésée, Paris, Ballard, 1677, acte II, scène 7.

(43) A. Rabillon, Notice di Ch.-S. Favart, P. Laujon, Parvi, Thésée, parodie, in Médée, un monstre sur scène. Réécritures parodiques du mythe 1727-1749, dir. I. Degauque, Montpellier, Éditions Espaces 34, 2008, p. 91.

(44) A. FrankLIN, Dictionnaire historique des arts, métiers et professions exercés dans Paris depuis le treizième siècle, Paris-Leipzig, H. Welter Éditeur, 1906 , p. 380.

(45) D. Roche, Histoire des choses banales. Naissance de la consommation XVII-XVIII siècle, $\mathrm{Pa}$ ris, Fayard, 1997, I partie, Ch. 3.

(46) «Les bouquetières parisiennes sont du même acabit que les marchands de poisson. Il est terrible de ne pas leur plaire: elles sont capables de vous jeter de la boue». J.P. Karamzine, Voyages en France, 1789-1790, citato da A. FARGE, Vivre dans la rue à Paris au XVIII siècle, Paris, Gallimard, 1992, p. 77. 
concesso da Luigi XIV ${ }^{47}$. Gli Écaillers nel repertorio forain non portano messaggi critici, appaiono strettamente connessi al gioco scenico e anche alla sua gratuità e in essi svanisce ogni riferimento all'attività economica; l'abito dei venditori di ostriche rientra infatti fra gli innumerevoli travestimenti adottati da Arlecchino per sfuggire e confondere chi lo perseguita, opponendosi al suo amore per Colombina. E questo rientra appieno nelle risorse sceniche ereditate dalla Commedia dell'Arte. Si assiste a delle gag di una grande semplicità: «[Arlequin] frappe à la porte. Pierrot l'ouvre; Arlequin lui dit qu'il apporte des huîtres que son Maître lui avoit commandées; Pierrot reçoit les huîtres, et pendant qu'il s'amuse à les examiner, Arlequin lui met un panier à la renverse sur la tête, et pendant qu'il se trouve ainsi aveuglé et embarassé, Arlequin se sauve dans la maison» ${ }^{48}$.

In Carolet, una Écaillère viene invece prescelta per impersonare parodicamente la nereide Teti e un coro danzante di Écaillères ${ }^{49}$ viene a sostituire il «Chœur de tous les dieux» con cui Fontenelle concludeva la tragédie lyrique musicata da Collasse, Thétis et Pélée $e^{50}$. L'ostricaia nutre seri dubbi sull'onestà del suo pretendente, un «capitaine de vaisseau» (che sostituisce il dio Nettuno dell'ipotesto) ${ }^{51}$ e gli preferisce un commerciante, «un petit bourgeois» (il corrispettivo di Peleo) ${ }^{52}$. La prosaicità delle situazioni e il punto di vista posto in basso, sostituiscono lo sviluppo lirico segnato dal soprannaturale in Fontenelle; una ricezione semplice può accogliere i significati evidenti proposti e rimanere appagata; lo spettatore più consapevole può operare il confronto con l'ipotesto e ridere anche della sfasatura operata nella riscrittura.

In un opéra-comique di anni successivi, un pescatore di nome Blaise parla e canta gioiosamente, affidando il suo pensiero alle metafore legate al suo mestiere e con questa risorsa riesce a consigliare all'innamorato infelice (Lubin-Pierrot) il modo per combattere il piano matrimoniale del gelosissimo tutore dell'amata: «On amorce le poisson I Pour qu'il entre dans la nasse. [...] Enlevez, enlevez, enlevez-la. I Dans ma barque je vous passe, | Enlevez» ${ }^{53}$. Con uno stile analogo e con metafore simili ma inasprite dalla satira, si esprimono i pescatori e le pescatrici in Arlequin déserteur di Louis Fuzelier: «Coquette aux yeux adroits et fins, | Pêchez de bons gros marsouins | Dans l'Océan de la finance, | Et zon etc., | C'est là qu'est l'abondance» ${ }^{54}$.

Fra gli altri mestieri svolti nella strada, Chaudronniers e Remouleurs entrano in scena per creare scompiglio. I calderai godevano di rispetto e nel XVI secolo erano inclusi nel «deuxième rang, qui sont les métiers entre les meilleurs et médiocres» ${ }^{55}$, costituivano una corporazione il cui primo statuto noto risale al 1327; trasformarono il loro lavoro in arte, decorando i catini fabbricati. Nel teatro forain vediamo i più

(47) A. Franklin, op. cit., p. 281. Dal 1690, vi furono in totale sei titolari dell'ufficio di «pourvoyeurs-vendeurs d'huîtres à l'écaille».

(48) Arlequin et Colombine captifs, ou L'Heureux désespoir, Divertissement Pantomime inventé et executé par le Sieur de Mainbray, et représenté sur le Théâtre des Sieur Restier et Veuve de La Vigne; à la Foire S. Germain, Paris, Gonichon, 1741, pp. 1213.

(49) D. Carolet, La Belle Ecaillere, Parodie burlesque de Thétis et Pélée (1736), BnF, ms. f. fr. 9315 , ff. $205 \mathrm{r}-226 \mathrm{v}$.

(50) B. DE Fontenelle, Thétis et Pélée, tragédie représentée pour la première fois par l'Académie Royale de Musique, le 1689 [sic] et remise au théatre avec des changements le 16 avril 1708, Paris, Ballard, 1708. Sulle parodie di questa tragedia in musica, cfr. F. Rubellin, Séductions et parodies dramatiques: Thétis et Pélée de Fontenelle et Collasse, in Littérature et séduction. Mélanges en l'bonneur de Laurent Versini, Paris, Klincksieck, 1997, pp. 675697.

(51) D. Carolet, La Belle Ecaillere cit., f. 208v.

(52) Ivi, f. 215v.

(53) J.-J. VadÉ, Le Poirier (1752), in Euvres de Vadé, Paris, Garnier, 1875, p. 182.

(54) L. Fuzelier, Arlequin déserteur (1715), in R. Guardenti, Le fiere del teatro. Percorsi del teatro forain del primo Settecento, Roma, Bulzoni, 1995, p. 229.

(55) Liste générale et rooles de tous les arts et mestiers qui sont en jurande, riportata da A. FRANKLIN, op. cit., pp. 211-212. 
umili fra questi artigiani, quelli della quinta classe, «chaudronniers à sifflet» che non avevano il diritto di esercitare il loro lavoro a Parigi e nelle grandi città. Percorrevano le campagne annunciandosi con un fischio e provvedevano alla stagnatura, alla riparazione e vendevano piccoli utensili di rame ${ }^{56}$. Per Arlecchino e Pierrot, fischiare e battere le pentole diventa immediatamente l'occasione per un gioco scenico che produce un crescendo gioioso di sonorità e movimenti ${ }^{57}$ : una libertà innocente che non rientrava ad esempio nella farsa composta all'epoca di Francesco I, in cui il protagonista calderaio fungeva unicamente da perturbatore dell'ordine familiare ${ }^{58}$.

Anche nel caso degli arrotini, che si erano uniti alla fine del XV secolo ai «couteliers» e formavano con essi la corporazione degli «esmouleurs de grandes forces» ${ }^{59}$, a teatro giungono i più modesti, quelli ambulanti chiamati anche gagne-petit e i cui gesti e suoni già il Théâtre Italien aveva messo in scena con effetti comici ${ }^{60}$. Savary descrive il «remouleur» con queste parole: «Pauvre compagnon coutelier, qui roule devant soi ou qui porte sur son dos une petite boutique garnie d'une meule, d'un marteau et d'une pierre à affiler» ${ }^{61}$. Arlecchino approfitta di questo travestimento per realizzare scherzi macabri con la mola e le spade che gli sono state affidate ${ }^{62}$ e Pierrot, nelle vesti di Remouleur, è protagonista di un'opera per marionette dove, mentre lavora, canta: «Braver l'insomnie | Dans un mauvais lit; | Or voilà la vie | D'un gagne-petit. [...] Je fais du remoulage | La plus fine fleur; | Et le plus fort ouvrage | Ne me fait point peur» ${ }^{63}$.

Lungo le vie offrono il loro servizio anche la Revendeuse e la Ravaudeuse; l'una vende abiti usati, l'altra rammenda quelli che le vengono portati e ha per bottega portatile una grande tinozza. Alla Foire Saint-Germain, una Colombina rammendatrice di calze da donna compare pronta a pungere con l'ago l'eterno spione Pierrot, per proteggere Arlecchino ${ }^{64}$.

Sebbene questi esempi mettano in luce in primo luogo il divertimento che nasceva grazie alle figure dei mestieri ambulanti in una drammaturgia comica, non possiamo pensare che negli artisti della Foire non vi fosse una coscienza della situazione reale delle persone che fungevano da ispiratrici. Essi stessi in buona parte avevano

(56) A proposito del lavoro dei «calderari», nella sua enciclopedica opera, La Piazza universale di tutte le professioni del mondo, e nobili ed ignobili (Venezia, Gio. Battista Somascho, 1585), Tommaso Garzoni scrive: «Nel principio, nel mezzo e nel fine i pezzi sono incommodi e spiacevoli a maneggiare. [...] Un valente maestro di tai lavori si scorge quando fa i lavori d'un pezzo giusto, uguale, per tutto sottile e ben garbato, senza molti colpi disordinati del martello [...]. E questi fabri ramari quando saldar gli occorre saldano con argento basso o con rame arso e borace, e bene spesso, anzi il più delle volte, con stagno e piombo mescolati; e con un poco di pece greca, e con un saldatoio di rame caldo [... . Presso agli antichi nella ramaria fu celebre Mamurio, onde Vertumno, presso a Properzio nel quarto libro, si gloria che il suo simulacro di rame fosse formato per mano di Mamurio». Grazie all'erudizione dell'autore e a una lessicografia ricchissima, è riconosciuta una dignità a tutti i mestieri, anche molto umili. L'opera ebbe più ristampe, una traduzione in tedesco, ma non in francese. Le edizioni moderne sono curate da Paolo Cerchi e Beatrice Collina (Torino, Einaudi, 1996, 2 voll.), da cui traiamo la citazione (pp. 739-740) e da Pina Di Meo e Luciano Carcereri (Firenze, Olschki, 1996).
(57) Arlequin Prothée par magie. Divertissement Pantomime; Inventé et executé par le Sieur de Mainbray. Et représenté pour la premiere fois sur le Théâtre des Sieur Restier et Veuve de Lavigne, à la Foire Saint Germain, le 3 Février 1741, Paris, Gonichon, 1741, p. 9.

(58) Farce nouvelle très bonne et fort joyeuse à trois personnages d'un Chauldronnier, in Le Théâtre Français avant la Renaissance: 1450-1550 cit., pp. 340-343.

(59) A. FrankLin, op. cit., pp. 618-619.

(60) J.-F. Regnard, Le Divorce (1688), in É. GHERARDI, Le Théâtre Italien, t. II, Paris, Briasson, 1741, p. 118.

(61) J. et L.-Ph. Savary Des Brulons, Dictionnaire universel de commerce (1723-1730), citato da A. Franklin, op cit., p. 619.

(62) J.-F. Colin, Arlequin tailleur par amour. Pantomime (1743), BnF, ms. f. fr. 9313, f. 268v.

(63) A.-R. Lesage, L. Fuzelier, J.-Ph. D'OrneVAL, Le Remouleur d'amour (1722), in A.-R. LESAGE, J.-Ph. D'Orneval, Le Théâtre de la Foire cit., t. V, p. 74.

(64) MaInBRAY, La Feste angloise ou le triomphe de l'bymen. Piéce pantomime, Paris, Gonichon, 1740, pp. 9-10. 
sperimentato il lavoro ultraprecario svolto all'aperto, le attrazioni e i numeri tradizionali offerti nei préaux delle fiere e nelle piazze, prima di inserirsi nelle imprese e nei luoghi più strutturati delle fiere stesse; erano per la loro storia parte di quel mondo dei margini, che veniva rappresentato. Come sottolinea Arlette Farge, «La rue parisienne à la fin de l'Ancien Régime est un espace que l'on ne choisit pas, un espace que l'on occupe pour la seule raison qu'on n'en possède guère d'autre, un espace pour vivre ${ }^{65}$. E da tale consapevolezza poteva nascere un teatro che voleva unire la cultura popolare a quella delle élites, i cui rappresentanti erano parimenti spettatori di queste opere.

Un posto non meno significativo in questi teatri ha la rappresentazione delle attività agricole e dei mestieri artigianali svolti al chiuso. Essa partecipa in qualche misura a un movimento molto più ampio, quello segnato dall'Encyclopédie, dove le attività manuali, gli strumenti e i termini che li indicano trovano uno spazio legittimo che ne evidenzia la necessità e il ruolo concreto nel progresso. Come evidenzia Maria Teresa Zanola, nel Settecento «le savoir technique ouvre les portes à la connaissance $\gg^{66}$ e Diderot auspica che dai laboratori e dalle fabbriche, l'esperienza venga assunta dagli scienziati e che l'innovazione sia sostenuta materialmente dagli uomini ricchi. Si tratta di un riscatto morale: «C'est aux Arts libéraux à tirer les Arts méchaniques de l'avilissement où le préjugé les a tenus si long-temps [...]. Les Artisans se sont crus méprisables, parce qu'on les a méprisés; apprenons-leur à mieux penser d'eux-mêmes: c'est le seul moyen d'en obtenir des productions plus parfaites» ${ }^{67}$.

Fra i lavori legati alla coltivazione e all'agricoltura, all'Opéra Comique non sono citati solo genericamente Paysans e Jardiniers, ben presenti nello spettacolo sin da epoche anteriori, ma sono rappresentate azioni specifiche come battere la canapa, zappare, rastrellare, fare covoni, far fascine, battere il burro, filare ${ }^{68}$. Alla battitura della canapa l'Encyclopédie dedica due tavole della sezione Agriculture et économie rustique, accompagnate da una dettagliata descrizione del processo di lavorazione, in cui sono nominati tutti gli attrezzi necessari; un'altra tavola riguarda la mietitura e il suo metodo.

Naturalmente, nel repertorio della Foire ricorrono le figure dei Vendangeurs, strettamente connesse con una filiazione popolare del tema bacchico; intorno ad esse ruotano anche delle canzoni, le cui strofe o timbres (versi iniziali) inneggiano a questo momento della vita rustica (celebre è Adieu paniers, vendanges sont faites). È Bacco in persona a spiegare ad Arlecchino come usare cesto e roncola nel vigneto e a fargli delle raccomandazioni: «Arlequin reçois de bon cœur | L'employ qu'on te destine, | Tu dois quand seras vendangeur | Epouser Colombine; | Va remplir un si beau destin, | Quelle sera ta gloire, | Si tu fais aussi bien le vin | Comme tu sçais le boire» ${ }^{69}$.

Un discorso a parte meritano i Bergers, i guardiani di buoi e i Fermiers, che per i loro caratteri definiti e non astratti avvicinano il tema pastorale alla realtà contemporanea, sottraendolo all'idealizzazione operata dalla letteratura, dalla musica e dalle arti figurative fino quasi a metà Settecento. Numerose sono le figure di ricchi borghesi possidenti terrieri. Uno di essi, Dorante, ha ricevuto dal re un incarico

(65) A. FARGE, op. cit, p. 19.

(66) M.T. Zanola, Arts et métiers au XVIII siècle. Études de terminologie diachronique, Paris, L'Harmattan, 2014, p. 44.

(67) D. DiDEROT, Art, in Encyclopédie ou dictionnaire raisonné des arts et des métiers par une société de gens de lettres (1751-1772), citato da M.T. ZANO-
LA, op. cit., p. 45

(68) F.-C. Boisard de Pontau, R.-B. Dourdet, L'Eil du maistre, Paris, Veuve Valleyre, 1742, p. 9.

(69) Arlequin et Scaramouche vendangeurs. Divertissement, Paris, s.e., 1711 (poi nell'ipertesto a cura di B. Russell, http://www.foires.univ-nantes. fr/play15.shtml). 
prestigioso ma fa queste riflessioni: «J'irois quitter une vie aisée et commode pour me livrer au tumulte d'une cour bruyante... Il n'en sera rien ${ }^{70}$; meglio una vita ritirata ma libera, lontana dalle costrizioni e dalle ipocrisie della corte. Ma i nobili di campagna che hanno figli, vogliono che questi si adeguino ai costumi di città, come M. d'Oysonvillain, «gentilhomme champenois» ${ }^{71}$. Dal canto loro, i Fermiers introducono nelle commedie il tema della vita economica, pensano a combinare matrimoni vantaggiosi per le loro figlie ${ }^{72}$; alcuni si chiamano come i pastori d'Arcadia, Tirsis, ma usano la canna da passeggio per minacciare i rivali ${ }^{7^{3}}$; altri sanno amministrare il loro patrimonio ${ }^{74}$. Il teatro della Foire intende mostrare delle piccole verità e con questo proposito giunge a riflettere le mutazioni sociali. Ma tiene conto anche dell'organizzazione apparentemente immobile della vita nei villaggi, riservando un posto ai Tabellions, notai di campagna, al Magister, maestro elementare, ai Prévôts e Maîtres de danse.

Anche il lavoro degli artigiani caratterizza molte scene e viene rappresentato nelle botteghe e negli ateliers, che costituiscono «l'antithèse de la rue dissipatrice» ${ }^{75}$, con attenzione e cura anche per gli strumenti e i gesti. Le figure degli artigiani rientrano nell'immaginario europeo e lo testimoniano numerose opere poetiche, narrative, musicali, coreografiche; la creazione dei forains si focalizza su alcuni dettagli concreti, segni di una condizione umana, seleziona tratti che suscitano il riso, senza giungere a uno studio psicologico, né a figure a tutto tondo, ma evita tanto le astrazioni quanto i ritratti estetizzanti.

Alla Foire appare più volte il ciabattino Blaise, a cui La Fontaine aveva dedicato un conte en vers ${ }^{76}$. Nelle vesti più nobili di «maître cordonnier», Blaise guida una bottega con vari garzoni, il cui tempo del lavoro è regolato dalla campana della refezione e difende il suo laboratorio da presunti ladri rincorrendoli sul tetto ${ }^{77}$. È evidente che non sono conservati qui né il carattere, né la situazione del conte. (Solo quattro calzolai potevano essere nominati maestri ogni anno, secondo lo statuto francese della corporazione, attiva sino al 1776). Nell'opéra-comique di Sedaine musicato da Philidor, vi è invece uno sviluppo abbastanza fedele al testo di La Fontaine; all'imbroglio comico puramente teatrale si unisce tuttavia un quadro realistico, Blaise «le savetier» è povero, non riesce a pagare l'affitto di casa e subisce il pignoramento dei mobili e del corredo della moglie $\mathrm{e}^{78}$. Un personaggio di Savetier povero e scherzoso era stato al centro di una farsa composta all'epoca di Luigi XII ${ }^{79}$. Nei libretti delle pièces realizzate attorno alla metà del Settecento trovano posto anche alcuni termini tecnici, come «tire-pied»e «alêne», il pedale e la lesina del calzolaio.

(70) F.-C. Boisard de Pontau, L'Art et la Nature. Ballet coupé de scenes (1737), BnF, ms. f. fr. 9338 , f. $109 \mathrm{v}$, in P. MARTINUZZI, Un matrimonio difficile: "L'Art et la Nature", balletto-pantomima inedito, «Ariel» XXIV, 1, 2009, p. 45.

(71) D. Carolet, L'Allure (1732), in A.-R. LesaGE, J.-Ph. D'ORneval, Le Théâtre de la Foire cit., t. IX, pp. 169-215.

(72) Roger, La Noce Angloise, «Mercure de France», août 1729, pp. 1844-1846; Ch.-F. PANNARD, Les Rivaux de village cit.; Ch.-S. FAvarT, Moulinet premier cit.

(73) L'Essai de la Folie, ou la naissance d'Arlequin. Divertissement Pantomime Représenté par la Troupe des Enfans Hollandois, Dans plusieurs des principales Villes et Cours de l'Europe, Liège, Everard Kints, [1742].

(74) F.-C. Boisard de Pontau, R.-B. Dourdet, L'Eil du maistre cit.; A.-R. LESAGE, L. FuZELIER, J.-
Ph. D'Orneval, L'Obstacle favorable (1728), in A.R. Lesage, J.-Ph. D'Orneval, Le Théâtre de la Foire cit., t. VI, pp. 257-312.

(75) Cfr. V. Milliot, Les Cris de Paris ou le peuple travesti. Les représentations des petits métiers parisiens (XVI'-XVIII siècles), Paris, Publications de la Sorbonne, 2014, p. 183.

(76) J. DE LA FONTAINE, Conte d'une chose arrivée à Château-Thierry (1699), in Fables, contes et nouvelles, éd. par J.-P. ColLINET, Paris, Gallimard, «Bibliothèque de la Pléiade», 1991, p. 417 sg.

(77) Mainbray, Le Diable boiteux. Divertissement Pantomime, s.1., s.e., 1743, pp. 11-12.

(78) M.-J. Sedaine, Blaise le savetier, opéracomique, suivi de La Nôce de Nicaise (1759), Paris, Duchesne, 1759.

(79) Farce nouvelle très bonne et fort joyeuse des Deux Savetiers, in Le Théâtre Français avant la Renaissance: 1450-1550 cit., pp. 210-215. 
Analogamente, i fabbri uniscono nella loro presenza scenica i gesti e i suoni della loro attività, l'immaginario popolare e la citazione colta: in una delle scene che li rappresenta, i Forgerons lavorano al servizio di Lustucru ${ }^{80}$, personaggio leggendario misogino chiamato anche «Opérateur céphalique» ${ }^{81}$, nella cui fucina si forgiano nuove teste per le donne dallo spirito ribelle e il cui motto è «Je racommode les langues à la façon du monde» ${ }^{82}$. In un'altra occasione, i fabbri lavorano per il dio Vulcano, mentre il pubblico canta questi versi: «Morbleu que l'on frappe tous I Si fort sur l'enclume, | Que la barbe en fume» ${ }^{83}$.

Fileuses, Brodeuses, Frotteurs, Maréchaux sono parte attiva, in quanto artigiani ma insieme agli artisti, nella dimostrazione del valore dell' $\left\langle\right.$ industrie» ${ }^{84}$, termine che per i forains riassume le varie accezioni evidenziate nell'Encyclopédie.

Quesnay définit l'industrie dans le sens de dextérité, d'adresse appliquées aux professions commerciales et artisanales. Dans un article de métaphysique l'industrie est une "faculté de l'âme dont l'objet roule sur la production et les opérations méchaniques; qui sont le fruit de l'invention, et non pas seulement de l'imitation, de l'adresse et de la routine, comme dans les ouvrages des artisans", et dans un article de droit politique et de commerce "ce mot signifie deux choses, ou le simple travail des mains, ou les inventions de l'esprit en machines utiles, relativement aux arts et aux métiers" ${ }^{85}$.

Lo spirito ricettivo e creativo che sceglie di mettere in scena alla Foire "arts et métiers" non può essere assimilato all'operazione contemporanea di stampa e diffusione dei Cris de Paris, non soltanto per l'interesse nutrito verso tutte le tipologie di occupazioni, più e meno qualificate, ma per l'incrocio di elementi popolari e colti, per l'assenza di un «discours sur le peuple, extérieur au peuple» ${ }^{86}$, di una logica moralizzatrice diffusa dalla seconda metà del XVII secolo. Secondo Daniel Roche, nella diffusione delle stampe, nella produzione poissarde e foraine si vuole cristallizzare, agli occhi del pubblico popolare, «un conflit dans l'imaginaire» ${ }^{87}$. Il conflitto certo esiste nella coscienza delle persone, e le pièces comiche della Foire non possono che metterlo in rilievo con un riso che non è sempre pacificatore, anche se è liberatore; va tuttavia tenuto conto anche dell'operazione poetica in sé, ed è questa che riesce ad amalgamare le istanze di una cultura popolare con quelle di una cultura consapevole degli sviluppi letterari e artistici, nel cammino delle idee, e in polemica con le forme irrigidite, garantite e tutelate da un organismo centrale e da un destinatario che apparteneva alla parte privilegiata della società d'Ancien Régime.

PAOLA MARTINUZZI

Università Ca' Foscari Venezia

(80) S.-J. Pellegrin, Arlequin Lustucru, Grand Turc et Télémaque (1715), BnF, ms. f. fr. 25480, ff. $108 \mathrm{r}-117 \mathrm{v}$, in P. MARTINUZZI, Le "pièces par écriteaux" nel teatro della Foire (1710-1715). Modi di una teatralità, Venezia, Libreria Editrice Cafoscarina, 2007, pp. 205-222.

(81) Stampa di Jacques Chiquet, datata tra la fine del XVII e l'inizio del XVIII secolo, Paris, Musée National des Arts et Traditions Populaires.

(82) Incisione di Esme de Boulonnois, 1657 circa, Paris, Musée National des Arts et Traditions Populaires.
(83) L'École des Jaloux, Paris, Valleyre, 1713 , p. 4 , in P. Martinuzzi, op. cit., p. 279. L'episodio è una parodia della prima scena de Les Adieux des Officiers di Charles Rivière Dufresny (1693).

(84) Ch.-F. Pannard, D. Carolet, L'Industrie, Balet mêlé de sçenes (1737), BnF, ms. f. fr. 9323, ff. 425r-428v.

(85) M. T. ZanOla, op. cit., p. 102.

(86) Così V. Milliot considera i Cris de Paris, op. cit., p. 189.

(87) D. Roche, La France des Lumières, Paris, Fayard, 1993, p. 298. 